\title{
Le relief des Landes du Mené et de leurs bordures occidentale et
} méridionale

Marcel Gautier

\section{Citer ce document / Cite this document :}

Gautier Marcel. Le relief des Landes du Mené et de leurs bordures occidentale et méridionale. In: Norois, $n^{\circ} 42$, Avril-Juin 1964. pp. 137-152;

doi : https://doi.org/10.3406/noroi.1964.1470

https://www.persee.fr/doc/noroi_0029-182x_1964_num_42_1_1470

Fichier pdf généré le 09/11/2019 


\title{
Le relief des Landes du Mené et de leurs bordures occidentale et méridionale
}

\author{
par Marcel GAUTIER
}

Le nom de Mené est un exemple de la persistance d'un toponyme breton en plein pays "gallo ". 11 a le sens de " montagne" (menez) et il désigne un ensemble de hautes terres dont l'élément majeur est constitué par la colline de Bel-Air $(340 \mathrm{~m})$ et par les "Landes du Mené ", un vocable maintenant très impropre puisque la lande en a presque disparu par le fait de la colonisation agricole entreprise depuis 80 ans. On retrouve le mot Mené dans les noms de SaintGilles-du-Mené et de Saint-Jacut-du-Mené, de même, vraisemblablement, que dans celui de Gommené. Dans l'acception locale, le nom de Mené s'étend à la région plus basse de Plessala-Langast, enfermée dans un cadre de hauteurs entre les Landes du Mené à l'Est et au Sud, les Landes de Phanton au Nord, la forêt de Loudéac à l'Ouest.

Le Mené constitue la limite N.-E. de la Bretagne centrale telle que je l'ai définie il y a plus de 15 ans. Depuis 1947, des faits nouveaux sont intervenus : apports récents des études géologiques, et notamment publication, en 1960, de la thèse de Mlle Durand sur le Tertiaire de Bretagne ; découverte et mise en exploitation, en 1958, d'une carrière de kaolin à Kerrouet, près de Saint-Gilles-duMené, à environ $250 \mathrm{~m}$ d'altitude, c'est-à-dire au voisinage même de la crête culminante des Landes du Mené, gisement qui, à notre connaissance, n'a pas encore été signalé. Il m'apparait donc nécessaire de reprendre l'étude morphologique de la région à la lumière de ces éléments nouveaux. A mon sens, c'est vers l'Ouest et vers le Sud des Landes du Mené qu'il faut rechercher les élé- 
ments de réponse aux problèmes que pose la morphologie de l'ensemble du Mené, d'autant plus que des éléments variés apparaissent dans ces deux directions : cuvette de Plémet, massif granitique de Gommené, glacis de la région de Merdrignac. Là apparaissent également des dépôts tertiaires qui permettent d'établir une chronologie des phénomènes. La présente étude englobe donc l'examen des hautes terres du Mené et de leurs bordures occidentale et méridionale (1).

\section{I. - LES LANDES DU MENE ET BEL-AIR}

Des abords de la Hutte à l'Anguille $(300 \mathrm{~m})$, l'on saisit nettement l'allure d'ensemble du Mené. La crête du Mené (fig. 1 et 5), orientée en gros NW-SE, dévie légèrement vers le Sud à la hauteur de Collinée, puis de nouveau sur le parallèle de la Hutte à l'Anguille. Elle se tient au voisinage de $300 \mathrm{~m}$ et les variations de son altitude sont médiocres, justiciables des seules modalités locales de l'érosion, sur la majeure partie de son tracé. Ce n'est qu'en approchant de Bel-Air que les cotes se relèvent, légèrement d'abord jusqu'à $314 \mathrm{~m}$, puis brusquement jusqu'à $340 \mathrm{~m}$. La crête domine au $\mathrm{N}$. et au N.-E. une région dont la morphologie est connue, et qui est constituée essentiellement par une surface de 100 à $120 \mathrm{~m}$, déformée autour du fond de la baie de Saint-Brieuc. L'on sait depuis longtemps que le Mené n'est pas un monadnock de résistance, puisque la crête se développe dans les schistes briovériens (ou schistes X), métamorphisés au voisinage de la granulite de SaintGilles. Le point culminant, Bcl- $\Lambda$ ir $(340 \mathrm{~m})$ est sur les schistes briovériens. Ce n'est pas non plus un monadnock de position. Sans doute, son relief en fait-il un château d'eau d'où les cours d'eau divergent. Mais Bel-Air n'est qu'à $21 \mathrm{~km}$ à vol d'oiseau du fond de la baie de Saint-Brieuc et le sommet le plus méridional (La CroixBouillard, $304 \mathrm{~m}$ ) qu'à $31 \mathrm{~km}$ de la mer. En outre, la transgression pliocène s'est avancée plus près encore d'un relief qui existait alors déjà. C'est donc du côté de la tectonique qu'il faut nous tourner pour expliquer son existence.

Du chemin qui s'en va vers le N.-E. en partant de la Hutte à l'Anguille, l'on saisit fort bien l'allure d'ensemble des hauteurs (fig. 2, coupe I). Vers le N.-E., la pente est forte, interrompue par un replat vers $240 \mathrm{~m}$. Vers le S.-W., une longue descente régulière s'amorce en pente douce, et l'on pense immédiatement à l'existence d'un bloc basculé vers le S.-W. G'est la même impression que produit Bel-Air vu du Nord, par exemple de la voie ferrée entre

(1) Carte au 1/200 000, édit. complète et tirage oro-hydrographique, feuille de Rennes, no $22 ; 1 / 100$ 000, tirage complet et tirage oro-hydrog., feuille de Loudéac ; $1 / 80000$ et cartes géologiques de Pontivy (no 74) et de St-Brieuc (2e édition) (no 59 ). 
Pommeret et Saint-Brieuc. Mais sur le front du bloc, près de BelAir, le replat se situe vers $260 \mathrm{~m}$ et non plus vers 240. Premier point : les Landes du Mené sont un bloc basculé vers le S.-W. ; le front du bloc est un escalier de failles, marqué par un gradin dont l'altitude varie de 240 à $260 \mathrm{~m}$. Ceci même indique des déformations plus complexes, dont la cote de Bel-Air constitue déjà une preuve.

Bel-Air se présente en effet comme un bloc surélevé de 30 à $35 \mathrm{~m}$, dont le revers s'accompagne d'un bloc annexe, orienté de la même manière et qui culmine aujourd'hui à $260 \mathrm{~m}$ (fig. 2 , coupe II). Il n'y a rien d'étonnant au fait qu'un effort tectonique plus intense se soit accompagné d'une plus grande complexité des ruptures du bâti et d'un parallélisme des déformations. Cette surélévation locale explique la dénivellation du gradin N.-E., déjà signalée, dénivellation qui suffit à montrer qu'il ne s'agit pas là d'un replat d'érosion postérieur à l'élaboration du relief. Or, comme on ne voit guère comment ce gradin serait antérieur au grand abrupt tectonique (faille I des figures), puisque rien ne lui correspond dans la zone littorale plus basse, force est de conclure à l'existence d'une marche de faille sur le front N.-E. du bloc basculé.

Mais vers le S.-W., les choses ce compliquent. Sans doute, la descente du bloc est-elle fort nette à partir de la Hutte à l'Anguille (fig. 1 et fig. 2, coupe I) ; elle est encore sensible à partir de BelAir (fig. 2, coupe II). Dans l'intervalle toutefois, la pente qui s'amorce à partir de la crête culminante des Landes du Mené s'interrompt vite. A des vallées séniles succède un encaissement rapide des cours d'eau, très sensible autour de Saint-Gilles-du-Mené. L'on dégringole vers une cuvette au fond accidenté, que l'érosion est bien loin encore d'avoir aplanie, et que nous appellerons cuvette de Plessala (fig. 3). Elle se développe dans des formations identiques à celles de la crête du Mené, schistes $\mathrm{X}$ et schistes métamorphiques.

\section{1. - La cuvette de plessala}

Elle se tient à l'altitude moyenne de $180-200 \mathrm{~m}$, accidentée de buttes et de vallées bien incisées. Au S.-W., un replat apparaît localement sur la rive droite de la vallée du Lié, replat que l'on peut raccorder " en pointillé » à la crête du Mené, en reconstituant un plan incliné qui est l'homologue de celui de la Hutte à l'Anguille. La cuvette interrompt donc la descente vers le S.-W. du bloc, et apparait ainsi comme un graben ouvert dans celui-ci. Vérs le N.-E., c'est par un abrupt qu'elle entre en contact avec les hautes terres du Mené, Au S.-W., sur la rive droite du Lié, la topographie a de nouveau l'allure d'un bloc basculé vers le S.-W. (fig. 3, coupe V). 


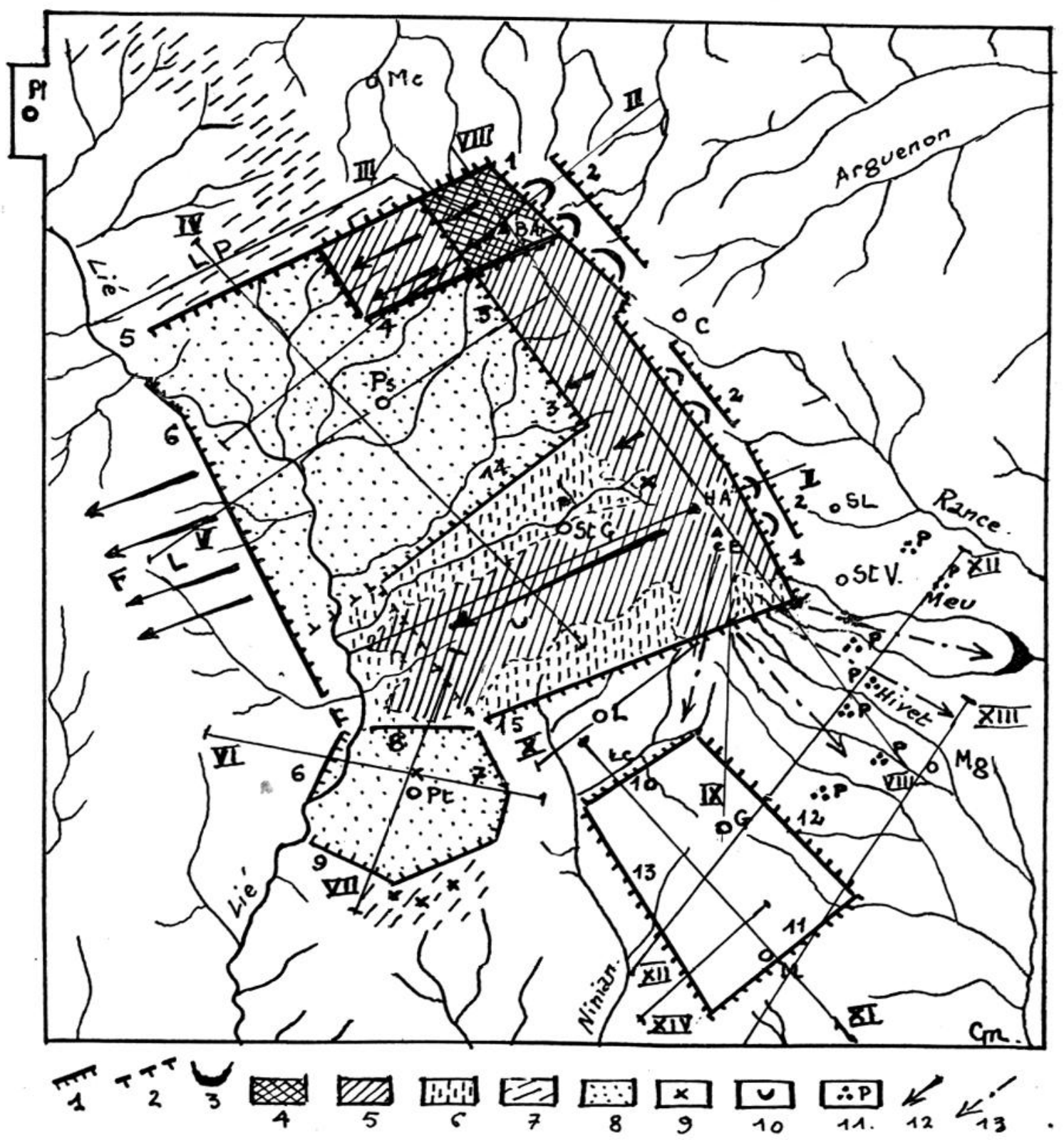

Fig. 1. - Morphologie des "Landes du Mené " et de leurs bordures occidentale et méridionale.

Légende : 1 : faille. - 2 : faille douteuse. - 3 :gradin de faille. -4 : bloc surélevé de Bel-Air. - 5: bloc basculé du Mené. - 6 : parties du bloc fortement ćrodées.7 : éléments hauts de la surface éogène. -8 : cuvettes tectoniques. - 9 : socène. 10 : carrière dans la roche profondément altérée. - 11 : pliocène. - 12: pente des blocs. - 13 : glacis méridjonal. - De I à XIV : tracé des coupes; les numéros des failles (en chiffres arabes) correspondent à ceux des coupes. Echelle : 1/200 000.

Pl : Plœuc ; Mc : Moncontour ; LP : Landes de Phanton ; BA : Bel-Air ; Ps : Plessala ; C : Collinée ; FL : Forêt de Loudéac ; StG : St-Gilles-du-Mené ; HA : I.a Hutte à l'Anguille ; CB: La Croix-Bouillard ; SL : Chapelle-St-Leu ; St V : St-Vran ; L : Laurenan ; Pt : Plémet ; LC : La Lande ès Chiens ; Mg : Merdrignac ; G : Gommené ; $M$ : Ménéac.

De toute évidence, les contacts sont ici tectoniques ct rien, dans la nature des roches, homogènes de part et d'autre des abrupts, ne peut les expliquer. Dans la direction perpendiculaire à celle-ci, 

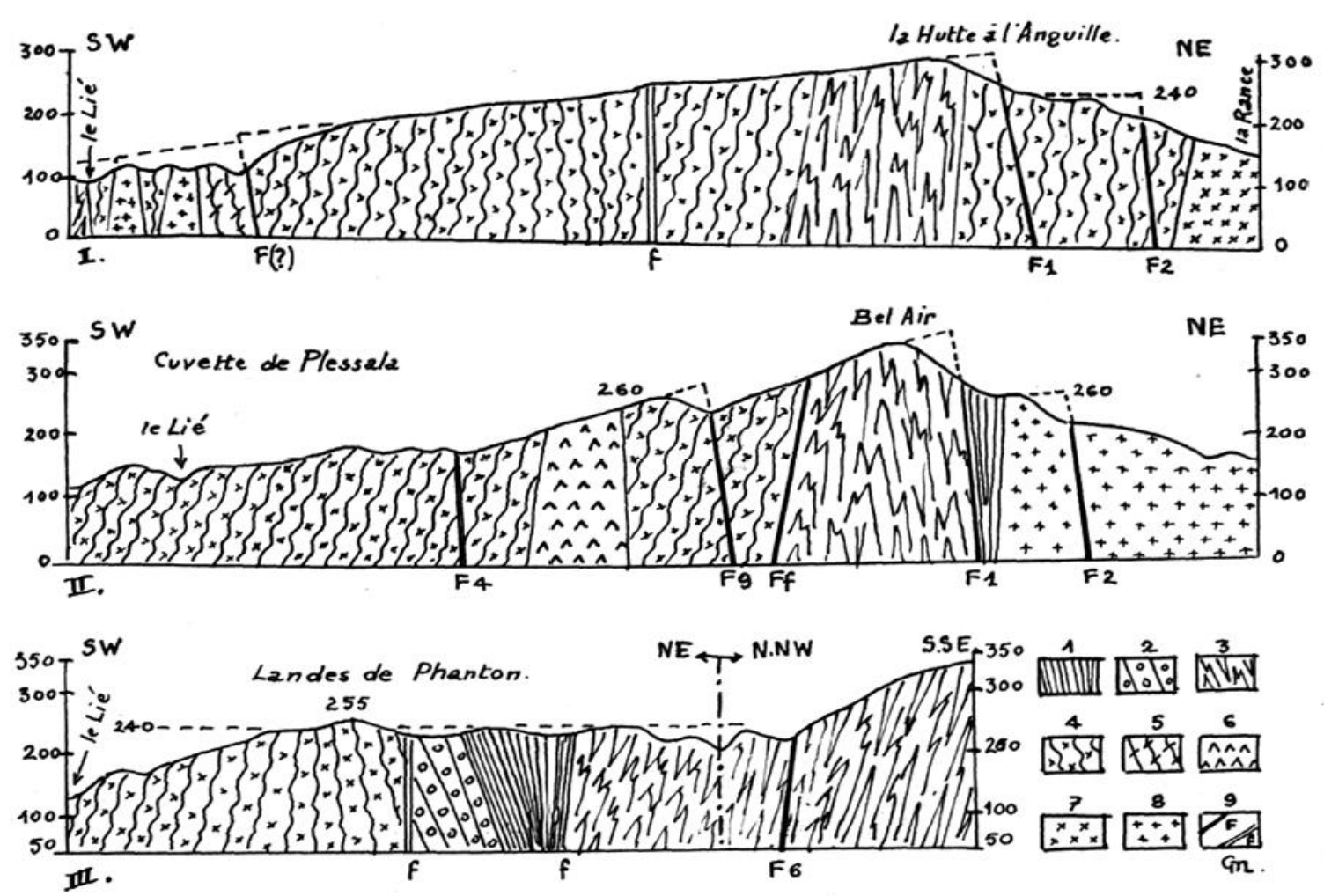

FIti. 2. - Le Mené et les Landes de Phanton.

Légende : 1 : dévonien. - 2 : silurien (grès armoricain). $-3:$ schistes briovériens ( $\mathrm{X}$ ). 4: micaschistes granulitiques. - 5 : micaschistes modifiés par le uranite. - 6.6 amphibolites. -7 : granulite. - $8:$ granite $-9: \mathrm{F}:$ faille tertiaire ; $f$ : faille primaire. Échelle des longueurs : $1 / 100000$. - Échelle des hauteurs : $1 / 10000$.
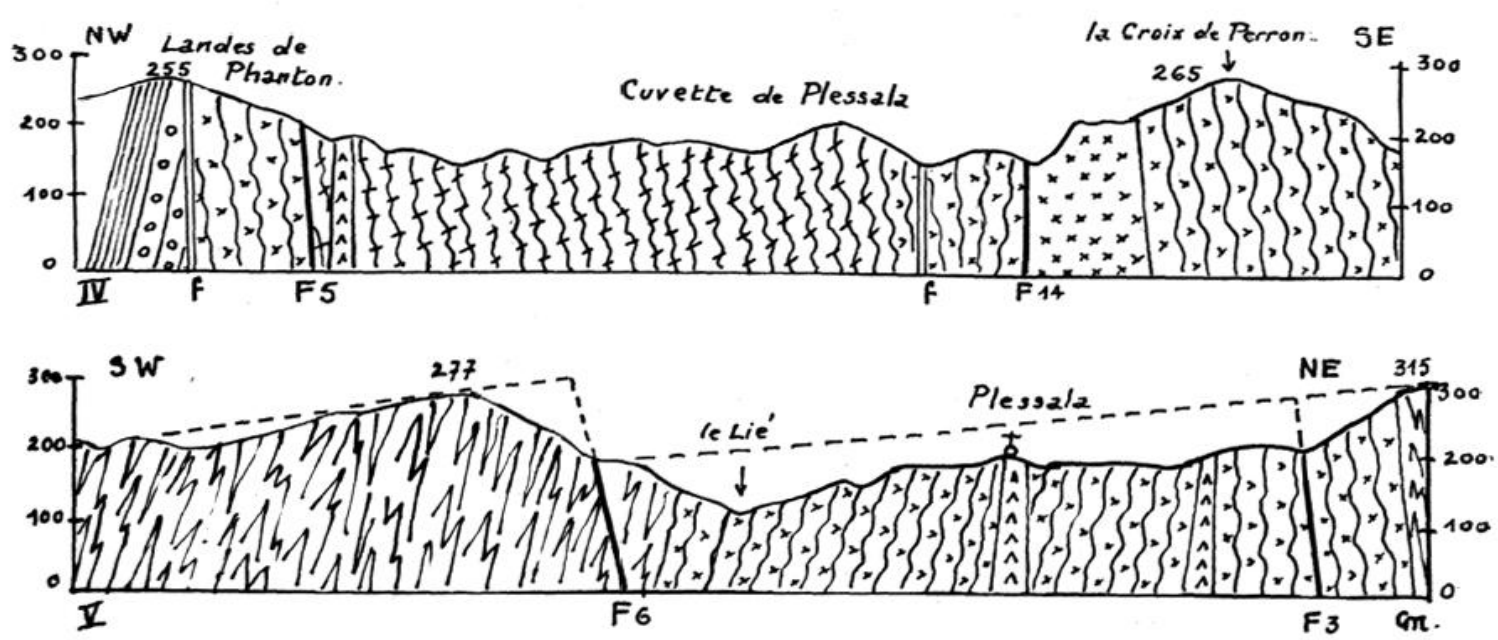

Fig. 3. - La cuvette de Plessala.

c'est-à-dire du N.-W. au S.-E., la cuvette paraît bien être également limitée par des failles. Vers le N., les Landes de Phanton tombent rapidement sur elle, alors que leur sommet amorce un niveau de hautes terres qui se retrouve au $N$. tout autour de la cuvette de Plœuc, jusque vers Saint-Carreuc, aux environs de 240- 
$250 \mathrm{~m}$. Éléments non déformés de la surface culminante des Landes du Mené (fig. 1 et fig. 2 , coupe III). Vers le S., le contact se confond localement avec l'apparition d'une amande granitique. Mais ailleurs, il s'opère en plein dans les schistes métamorphisés. Une faille limite visiblement là le revers du bloc qui descend de la Hutte à l'Anguille. Mais elle est masquée, comme elle l'est plus au Sud, de l'autre côté de la longue pente NE-SW, par les attaques de l'érosion sur les bordures du grand plan incliné (fig. 1). A mon sens, c'est entre le ruisseau de Saint-Gilles ct celui de SaintGouéno que doit se situer l'accident tectonique, et non au Sud du premier, à l'aplomb mêrme de la longue pente actuelle. Sinon, le relief disséqué des environs de Saint-Gilles ne recevrait pas d'explication. Or il représente, à notre avis, ce qui subsiste du démantèlement du revers du bloc vers l'amont des petits cours d'eau.

Ainsi, la cuvette de Plessala apparait bien comme un quadrilatère effondré dans le revers du bloc basculé des Landes du Mené. L'érosion différentielle ne l'explique pas. Au reste, l'on saisirait mal comment le Lié, qui l'évite et se borne à longer sa bordure occidentale, aurait pu la déblayer avec l'aide de ses affluents alors que sa vallée s'étrangle à la sortie aval. Par contre, la disposition même du réseau hydrographique milite en faveur de l'existence d'une cuvette tectonique : ruisseaux courts sur la rive droite du Lié, c'est-à-dire sur le front du bloc basculé occidental ; ruisseaux plus longs sur la rive gauche, à l'intérieur de la cuvette.

\section{III. - LA BORDURE MERIDIONALE}

Elle est caractérisée, en dehors de la zone de dissection du plan incliné déjà signalée, par trois types de reliefs : le horst granitique de Gommené au centre, la cuvette tectonique de Plémet à l'Ouest, un glacis à l'Est.

La région granitique de Gommené (fig. 1 et 6 ) pourrait tenir de sa nature même son altitude légèrement plus forte que celle des régions encadrantes. Elle atteint $223 \mathrm{~m}$, dominant ainsi de 50 à $70 \mathrm{~m}$ en son centre les contrées voisines. Mais le granite ne correspond pas nécessairement aux parties les plus hautes au Nord et le glacis dont il sera question plus loin mord sur lui. Au S.-E. de Ménéac, la rupture de pente est en plein granite. A la notion de bloc résistant, l'on doit donc substituer celle d'un horst. Au N.-W. toutefois, la structure se complique. Un ruisseau rectiligne traverse la région marécageuse de la Lande ès Chiens, en bordure de la route nationale 164 bis de Loudéac à Rennes. A un écoulement N.-S. se substitue localement, sur environ $3 \mathrm{~km}$, un écoulement N.E-SW (fig. 6, coupe XI). II paraît bien marquer la limite septentrionale du horst de Gommené. Dans cette région Nord, la tranchée ouverte 
pour la rectification du tracé de la $R$. N. près du Hambo (2) a d'ailleurs fait apparaître la grande irrégularité du contact entre le granite et les micaschistes très décomposés. La carte géologique simplifie là une réalité très complexe (3).

A l'Ouest du horst, entre le revers du bloc basculé du Mené et la surface qui se développe à l'altitude de $180 \mathrm{~m}$ au Nord de la Ferrière, s'ouvre la cuvette de Plémet-dont le Lié longe le rebord
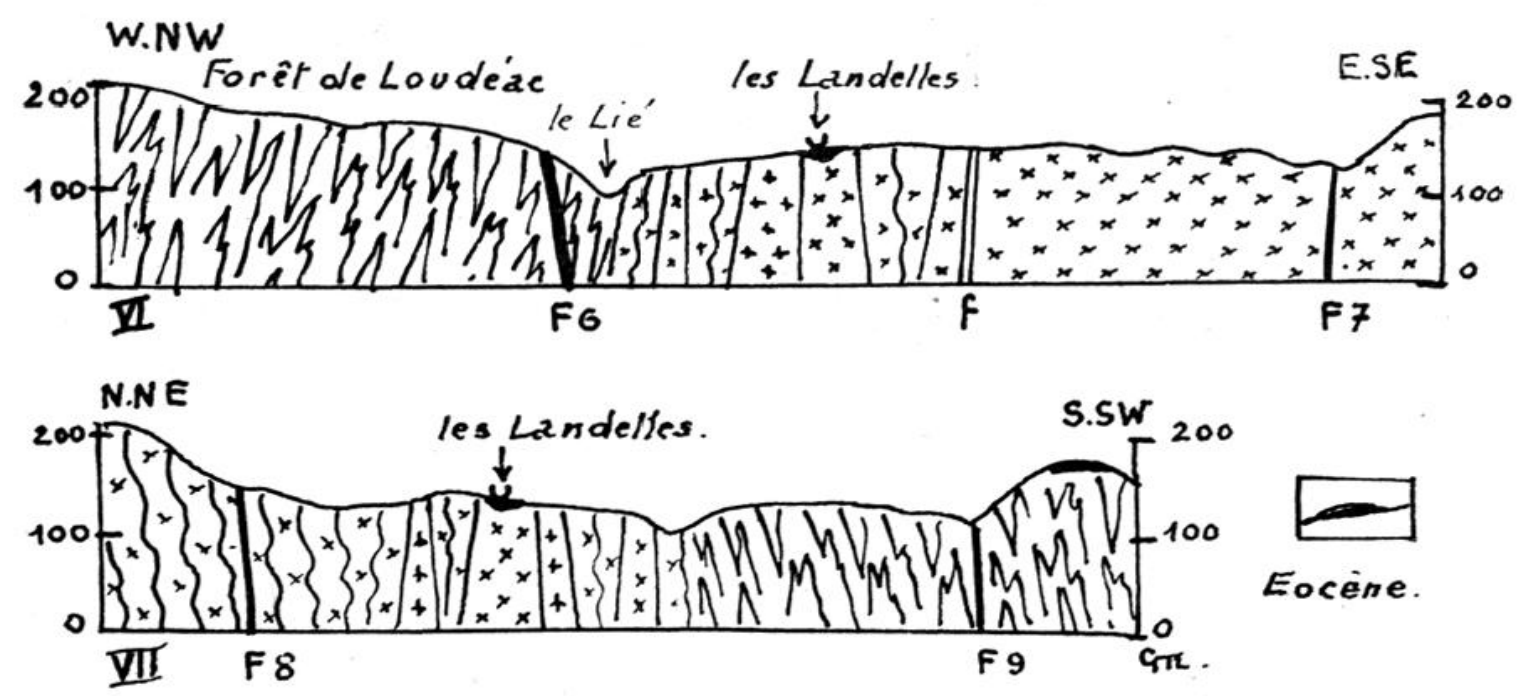

Fig. 1. - La cuvette de Plémet.

occidental comme il le fait de la cuvette de Plessala. Là aussi, l'origine tectonique de la dépression n'est pas douteuse, même abstraction faite des dépôts que l'on trouve dans son fond et sur son rebord méridional, dépôts sur lesquels nous reviendrons. En effet (fig. 4), les flancs de la cuvette sont modelés dans des formations analogues à celles du fond : schistes $X$ au N.-W. et au S.-W., schistes métamorphisés au N.-E., granulite au S.-E. Et puisque le Lié et ses affluents l'évitent, ou presque, en se bornant à suivre ses lisières, il ne reste que l'explication tectonique pour justifier de l'existence de la dépression.

Plus énigmatique est le glacis qui s'incline doucement de SaintVran à Merdrignac, au N.-E. du horst de Gommené (fig. 1). Tout un éventail de cours d'eau s'ouvre au-dessous de la Croix-Bouillard. Vers le S.-W., le glacis avorte et s'interrompt vite au contact du horst de Gommené (fig. 5, coupe 1X). Mais vers le S.-E., il se développe sur plus de $10 \mathrm{~km}$ pour venir se raccorder à un niveau de

(2) Suppression du “ virage des Américains », ainsi nommé en raison des nombreux accidents survenus là à des motocyclistes américains, débarqués à Brest, pendant la guerre 1914-18.

(3) L'on peut noter que les failles anciennes, figurées sur la carte géologique, ont rarement rejoué au Tertiaire. L'on ne peut guère citer, comme exemple de ce rejeu, que celui de la faille Nord du Mené (no 5 de la fig. 1). 
$100 \mathrm{~m}$ d'altitude moyenne, largement étendu. La descente s'amorce vers $240 \mathrm{~m}$, au contact de l'abrupt estompé que détermine la faille limite du bloc basculé des Landes du Mené (faille 15, fig. 1 et 5). S'agit-il donc, là encore, d'un bloc basculé ? Originellement,
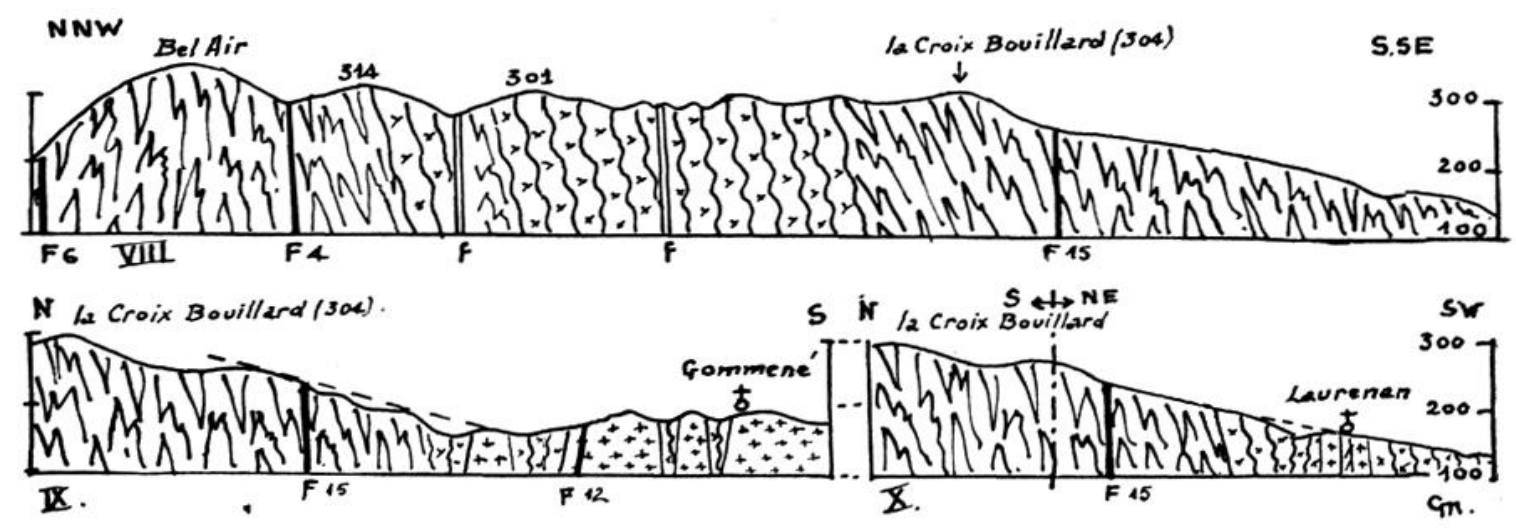

Fici. 5. - La crête et le glacis méridional du Mené.


Fic; 6. - Allure transversale du glacis et relations avec le horst de Gommeni.

sans doute ; encore qu'il soit à peu près impossible d'en localiser les failles limites en dehors de celle qui vient d'être mentionnée. Tout au plus peut-on faire observer, d'une part, que la région de la Chapelle-Saint-Leu, à l'E-NE de la Croix-Bouillard, a pu être interprétée comme un horst à la limite du glacis (4), et que la faille limitant au N.-E. le horst de Gommené pourrait être en même temps l'accident le long duquel aurait joué le bloc basculé du glacis.

(4) G. FLEL RY : Le relief ef le réseau hydrographique de la région de la haule Hance. du Meu et de l'Hivel (Diplôme d'F.S., Rennes, 1950 ; un exemplajre dactyloụ. conservé au Labo. de géoğ. de la Faculté des Lettres de Rennes). 
Mais au N.-W. et au S.-E., l'on ne trouve rien. L'explication tectonique est plausible, sans être entièrement démontrable. Par ailleurs, la surface topographique, transversalement multiconvexe dans ses détails (fig. 6), n'est pas une surface conique et le parallélisme des cours d'eau aux environs de Merdrignac, cours d'eau non orientés selon les génératrices d'un cône, l'indique suffisamment. En outre, cette surface porte des dépôts tertiaires dont la datation reste controversée. C'est donc sur ceux-ci qu'il faut nous pencher pour tenter d'élucider cette forme topographique singulière, ce qui nous conduit à l'examen de l'ensemble des formations tertiaires dans la région étudiée, fondement d'un essai de chronologie des phénomènes.

\section{IV. - CHRONOLOGIE DES PHÉNOMÉNES}

J'avais, en 1947, considéré le Mené comme un élément de la surface supérieure de M. R. Musset, affecté d'un basculement antérieurement à l'Éocène. Et la zone de Bel-Air m'avait paru correspondre à un axe de soulèvement maximal. En 1952, M. S. Martin voyait également dans le Mené un bloc basculé, dont le soulèvement aurait " commencé assez longtemps avant l'achèvement de la surface éocène ". Il voyait par ailleurs, dans les gradins à 240$260 \mathrm{~m}$ du front du bloc (N.-E.), des replats d'érosion élaborés au cours du soulèvement, par achèvement définitif du cycle qui aurait réalisé la surface culminante (5). J'ai donné plus haut les raisons qui me font écarter ce point de vue et voir dans ces gradins les éléments d'un escalier de failles. Et je pense maintenant que le bloc du Mené représente tout simplement un élément de la surface éogène, basculé au Miocène. Ceci, du fait de la découverte, en bordure de la route de Saint-Vran à Saint-Gouéno qui longe la crête au S.-W., d'une carrière de kaolin mise en exploitation en 1958, près du hameau de Kerrouet, à $250 \mathrm{~m}$ environ d'altitude. En fonction, d'une part, de l'existence sur le Mené de roches profondément altérées.

Sans doute n'est-il pas impossible que la "maladie tertiaire" des terrains bretons (6) ait affecté des surfaces topographiquement dénivelées. Les arènes granitiques épaisses de $15 \mathrm{~m}$ et plus, les schistes briovériens et métamorphiques décomposés sur une grande épaisseur et dont les argiles de décomposition prennent parfois une teinte groseille caractéristique, les concrétions et les dépôts sidérolithiques, les grès ladères, dits grès à Sabals, se rencontrent ailleurs dans la région et bien loin d'elle dans la péninsule armoricaine. Mais

(5) S. Martin : Essai d'interprétation morphologique des Monts du Mené et des régions avoisinantes (Bull. A.G.F., 1952, pp. 37-43).

(6) L'expression est de M. Y. Milon, dont point n'est besoin de rappeler ici les travaux sur les formations tertiaires de Bretagne. 
si Mlle Durand (7) ne conclut pas sur les différences d'altitude entre les surfaces qui portent du Sidérolithique, surfaces qui atteignent $170 \mathrm{~m}$ à Plémet, plus de 200 sur les Landes du Mené, 95 $\mathrm{m}$ à l'Ouest de Lamballe et beaucoup moins dans la dépression du Gouessant, elle considère qu'elles appartiennent à un relief différencié dès l'Éocène et incomplètement aplani, ou bien que leur dénivellation résulte de l'action des phénomènes tectoniques reconnus au cours du Tertiaire. Elle ajoute (p. 133), que s'il vaut mieux parler d'une topographie ou d'une surface, plutôt que d'une pénéplaine éocène, "le relief devait être assez adouci sous la couverture de roches altérćcs ". Or, la crête des Landes du Mené a un commandement de $100 \mathrm{~m}$ au moins ; ce qui ne correspond pas, à l'échelle des reliefs bretons, à une topographie " assez adoucie ".

En outre, l'on comprendrait mal comment des argiles n'auraient pas flué sur les pentes et se seraient conservées, comme celles qui furent exploitées durant un temps près du carrefour de la route du Vaublanc et de la route de Saint-Gilles à Plémet, vers $240 \mathrm{~m}$ d'altitude, argiles qui témoignent d'une altération sur place puisqu'elles conservent l'empreinte du feuilletage des micaschistes et que la structure de la roche mère n'est pas perturbée (8). Argiles en place, sur la pente même du glacis qui les invitait à couler vers le bas par gravité (fig. 1). Force est donc d'admettre qu'à l'Éocène, la pente n'existait pas.

En effet, les hypothèses possibles sont les suivantes : ou bien la " maladie tertiaire " se développa sur des surfaces d'érosion étagées à 300, 180-220, 100-120 m d'altitude moyenne; ou bien elle se développa sur un glacis préexistant, en vertu d'un mouvement de bascule antérieur à l'Éocène ; ou bien ce mouvement est postérieur à l'Éocène. Dans le premier cas, l'on observerait une curieuse coïncidence entre les limites du bloc basculé et celles du haut niveau antérieur sur 3 des faces du bloc. Il paraît donc plus plausible de considérer le Mené comme un horst basculé affectant une surface unique, ce qui présente l'avantage de supprimer une coïncidence providentielle. Dans le deuxième cas (mouvement de bascule antérieur à l'Éocène, solution que j'avais envisagée en 1947), la a maladie tertiaire " se serait développée sur un relief préfigurant le relief actuel, sauf quelques retouches ultérieures. Mais cette explication conduit ici à reculer dans un passé géologique lointain, indéterminé, des déformations qu'il est plus normal de rattacher aux dislocations miocènes, dont l'extension en Bretagne est à peu près générale et dont la datation a pu être établie en maints endroits. Au reste, je considère aujourd'hui que la tendance à vieillir les reliefs bretons a parfois été excessive.

(7) Mile S. Durand : Le Tertiaire de Bretagne (Thèse Sc. nat. Rennes, 1960, Mémoires de la Soc. géol. el minéral. de Bret., t. XII, 1960, 1 I I + 389 p.), p. 148.

(8) Id., p. 128. 
L'on peut, en outre, se demander si l'intense décomposition des schistes du Mené ne se serait pas accompagnée à l'Éocène, alors que l'attaque des roches était très avancée, d'une descente en coulées des épais dépôts sur les pentes lors des périodes de forte pluviosité. Ce qui n'expliquerait pas la conservation de la structure des schistes dans les argiles de décomposition. Sans doute, les climats qui régnèrent sur la région au Miocène et jusqu'au Pontien inclus, voire au Pliocène, firent-ils alterner phases chaudes et humides et phases chaudes et sèches. Mais les températures décroissaient et l'altération des roches fut moins intense au Pliocène qu'à l'Éocène (9). Durant certaines périodes, les conditions d'une descente des for mations molles sur les pentes furent réalisées. Par ailleurs, le cl imat périglaciaire du début du Quaternaire a permis, en Bretagne, le glissement des coulées de head sur le flanc de maints versants. L'on pourrait donc en conclure que le maintien sur place des formations molles de l'Éocène ne prouve pas ici la postériorité des déformations tectoniques. Mais autre chose est l'existence de terrains de décomposition épais et visqueux sous le climat qui les engendre, autre chose la possibilité de leur mise en mouvement après leur consolidation relative. L'érosion pliocène s'est exercée sur la couche superficielle de ces formations, et les coulées boueuses périglaciaires n'ont également affecté que leurs couches superficielles, au-dessus d'un pergélisol résistant. Dans les deux cas, la descente n'a pas pu affecter la masse, relativement durcie, des argiles de décomposition. Je pense done qu'il est difficile de dater autrement que du Miocène les déformations tectoniques du Mené.

Quant au kaolin de Kerrouet, il est également " en place " et il a été formé par altération, au contact de la granulite de Saint-Gilles et des schistes métamorphiques voisins. La couche exploitable est épaisse d'environ $30 \mathrm{~m}$ (10). Le gisement est donc analogue à celui de Plémet dans la genèse duquel ont joué des facteurs internes. Les kaolins de Bretagne sont d'ordinaire datés de l'Éocène. On ne les connaissait jusqu'alors que dans les régions déprimées, soit sur le littoral de Plœmeur près de Lorient, soit dans la région de Quessoy, sur la surface de 100 à $120 \mathrm{~m}$, soit dans la cuvette tectonique de Plémet. Leur présence au voisinage de la crête culminante du Mené permet, conjointement aux arguments tirés des argiles de décomposition des schistes, de conclure que le Mené est un élément de la surface éogène, et que la surrection et le basculement du bloc sont un des aspects des mouvements reconnus en Bretagne au Miocène.

(9) Sur les paléoclimats dans l'Ouest, cf. M me Ters : La Vendée littorale, Thèse, 1962, passim; sur la décomposition moins intense des roches au Pliocène, cf. Mile S. Durand, Thèse, p. 321 .

(10) Renseignements aimablement communiqués par $M$. le Directeur des usines de la Soc. anonyme des kaolins et produits réfractaires de Bretagne, à Plémet, le 22 nov. 1963. L'extraction est d'environ 1.200 tonnes par mois. 
Miocène, également, la formation de la cuvette de Plémet, dont les grès ladères et les formations sidérolithiques $\mathrm{du}$ fond se retrouvent sur son bord méridional, dans la région de la Ferrière. Et l'on peut, en extrapolant faute de dépôts, dater également du Miocène le horst de Gommené, ainsi que la cuvette de Plessala et le bloc basculé à l'Ouest du Lié. La surface éogène a donc subi de notables distorsions dans la Bretagne centrale, puisque si on la trouve à $300 \mathrm{~m}$ et plus dans le Mené, à 250-255 m autour de la cuvette de Plœuc, elle n'est plus qu'à 100-120 m dans la zone littorale entre Saint-Brieuc et Dinan, où des grès ladères et des lambeaux de formations sidérolithiques permettent de la dater.

L'on pourrait objecter que les fonds de cuvettes, de Plessala à Langonnet, au pied de la Montagne Noire, sur les confins du Finistère et du Morbihan, à près de $100 \mathrm{~km}$ de distance, en passant par Saint-Tugdual, se tiennent d'une façon assez troublante au voisinage des cotes $180-200 \mathrm{~m}$. Mais celle de Plémet est légèrement plus basse. De même, la surface éogène rejoint, dans la région littorale au Nord du Mené, les cotes de la surface dite du Léon. Les horsts eux-mêmes présentent une relative analogie d'altitude : $300 \mathrm{~m}$ pour les Landes du Mené, mise à part la zone surélevée de Bel-Air ; $289 \mathrm{~m}$ pour la Montagne de Locronan, $284 \mathrm{~m}$ pour le horst de Mellionnec. Mais ces correspondances ne sont qu'approchées, et elles souffrent des exceptions. Comme il est difficile d'imaginer l'excavation d'une cuvette par un cycle d'érosion normale lorsque la dépression n'offre pas de conditions lithologiques différentes de celles de son cadre, qu'elle est d'ampleur réduite et ne s'ouvre à l'aval que par une brèche étroite, l'on peut penser que les concordances d'altitude, encore une fois assez approchées, signalées plus haut, peuvent s'expliquer par le seul jeu des facteurs tectoniques. Des forces d'intensité sensiblement analogue ont dû provoquer des effondrements et des surrections d'une ampleur assez uniforme en des points qui ne sont éloignés les uns des autres qu'à l'échelle d'une province. Sans pour autant, d'ailleurs, négliger les possibilités de remaniements ultérieurs des fonds de cuvettes et des reliefs par des phénomènes érosifs. L'inégalité du plancher de la cuvette de Plessala montre que ces remaniements sont restés inachevés.

Reste le problème du glacis de Merdrignac. On a pu (11) l'interpréter comme étant un pediment, en se référant à des observations faites antérieurement sur d'autres points du Massif armoricain (12). Encore que l'on ait trouvé la pente du glacis faible pour un pedi-

(11) G. FleURY, op. cit.

(12) Hug. MU RY : Aspects physiques des confins normands bretons (Chronique géog. des Pays reltes, Ann. de Bretagne, 1950, Rennes, 1951, pp. 1 a 6). A. Nif.YniEr : A propos des pediments armoricains (Chr. géog. des Pays celtes, Ann. de Bret., 1952, fasc. 2, pp. 127-128). 
ment et que l'on n'ait pu déceler de talus amont ; l'on a donc supposé celui-ci nivelé par l'érosion ultérieure, la Croix-Bouillard et la cote 273, au Sud de celle-ci, représentant les restes d'inselbergs après nivellement du talus. Pour ma part, je ne pense pas qu'il y ait là le moindre vestige d'inselberg, mais bien des éléments du bloc basculé du Mené. Les témoins du talus ne sont peut-être pas totalement absents si l'on y regarde de près (fig. 5). Mais il est certain que la pente est bien faible pour un pediment (13). Elle n'est ici, en moyenne, que celle qu'il est possible de trouver vers l'aval d'une telle forme et légèrement supérieure à $1 \%(1,2$ à 1,4$)$. L'absence d'un knick véritablc à l'amont pourrait aisément résulter de l'érosion ultérieure dans des schistes déjà relativement peu résistants quand ils sont sains, et par surcroît très altérés par la "maladie tertiaire ". Le Mené quaternaire n'évolua pas dans un régime d'aridité ! En outre, dans une Bretagne où les coulées de head témoignent d'actions périglaciaires non négligeables, les fortes pentes du Mené ne durent pas rester en dehors du domaine de la solifluction. De fait, le head est ici présent. Le glacis lui-même peut vraisemblablement hériter d'un bloc basculé dont l'existence est discutée plus haut. La faiblesse de la pente résulte-t-elle de la décomposition des schistes qui auraient offert à l'écoulement en nappe un matériel particulièrement vulnérable, ou d'un relèvement ultérieur de la surface, pivotant tout entière autour de l'axe tectonique ayant guidé le knick, et en sens inverse du premier mouvement? La question reste posée. D'autant plus que le glacis porte des dépôts dont la date est encore mal établie.

Du hameau de la Bourdonnais au Nord, au hameau de Castelnouët au Sud, par le Rancelais, le Bois de Saint-Vran, les Forges de la Hardouinais, Launay Trébédé, la Grionnais, sur la R.N. 164 bis, et jusqu'à l'altitude de $170 \mathrm{~m}$, l'on trouve des gisements de galets et de poudingues que j'avais, en 1947, datés de l'Éocène. L. Aufrère, en 1928, faisait de Merdrignac. "le centre du faux Pliocène ", où le "faux Pliocène est peut-être éocène " (14). M1lë S. Durand, suivie par G. Fleury, pense, par contre, que les minerais de Castelnouët " ressemblent à du véritable Pliocène ", en raison du fait qu'ils sont "identiques aux roussards, si fréquents à la base des sables rouges armoricains ", lesquels sont pliocènes (p. 127). Mais elle ajoute (p. 315) que les " poudingues à galets blancs de Castelnouët sont peut-être pliocènes. "Peut-être éocènes ", " peut-être pliocènes ", voilà bien des " peut-être " sous la plume des géologues. Toutefois, Mlle Durand fait observer que les poudingues renferment là des galets de grès lustrés éocènes. L'argument est de poids. Il

(13) J. Dresch : Pediments et glacis d'érosion, pediplains et inselbergs. (L'Information géographique, 1957, pp. 183-196).

(14) L. Aufrère: Recherches en Bretagne, Épreuves et synthèses, no 3, Paris, 1938, pp. 74-79. 
prouve le remaniement de dépôts éocènes du glacis et leur incorporation dans des poudingues présumés pliocènes. Ces cailloux roulés ont subi un transport, indiqué par leur morphologie même. De quelle longueur et de quelle nature ? Le glacis est bien court pour permettre la formation de "galets " de quartz et de grès quartzites par un écoulement boueux ou semi-concentré. Alors, un façonnement marin ? C'est la solution vers laquelle pencha G. Fleury après avoir calculé l'indice d'aplatissement, l'indice de dissymétrie et l'indice d'émoussé de 60 d'entre eux pris au hasard, selon les méthodes d'A. Cailleux. D'après ces mensurations, $50 \%$ des galets examinés présenteraient un indice d'aplatissement qui indiquerait une origine marine, 25 à $27,5 \%$ un indice de dissymétrie, $60 \%$ un indice d'émoussé conduisant à la même conclusion. Les histogrammes concernant l'indice d'émoussé des galets des Forges et de Castelnouët, établis par G. Fleury, diffèrent sensiblement d'allure. Pour les Forges de la Hardouinais, l'histogramme se rapproche de celui donné par J. Tricart et $R$. Schaeffer pour les galets de quartz à usure marine (15). Celui des galets de Castelnouët s'en écarte davantage. Aux Forges, 40 galets ont été étudiés. A Castelnouët, 20 seulement. Cet écart du simple au double est peut-être responsable de cette particularité. Mais en tout état de cause, le nombre des mensurations fut beaucoup trop faible pour donner des résultats qui permettent de conclure. Les analyses de G. Fleury n'autorisent que des présomptions.

Quoi qu'il en soit, il n'est pas interdit d'envisager l'hypothèse d'une transgression pliocène ayant atteint localement l'altitude de $170 \mathrm{~m}$, sous l'influence d'une déformation de la surface topographique. Mlle S. Durand envisage (p. 332) " un nouveau jeu des voussoirs " accompagnant la transgression pliocène. M. A. Meynier a décelé, en Bretagne, des accidents tectoniques pliocènes (16). Et j'ai, pour ma part, suffisamment admis ailleurs l'existence de mouvements pliocènes, ou même postpliocènes, en Bretagne pour ne pas refuser ici l'explication.

Il n'en reste pas moins que la question ne deviendra claire qu'après dissipation de l'incertitude qui persiste au sujet de l'origine et de l'âge de ces galets et des formations qui les accompagnent. Provisoirement, et en acceptant la datation " pliocène ", l'on pourrait schématiser ainsi l'évolution du glacis : préfiguration de celuici par un bloc basculé miocène ; développement d'un pediment dans l'hypothèse "continentale ", qui est la plus simple sans être pleinement satisfaisante ; dans l'hypothèse " littorale ", élabora-

(15) J. Tricart et R. Schaeffer : L'indice d'émoussé des galets, moyen d'étude des systèmes d'érosion. (Revue de géomorphologie dynamique, 1950 , 1 re année, n' 4, pp. $151-179$; fig. $6 \mathrm{~B}$, p. 163.)

(16) Voir, notamment : "Une faille pliocène ?" (Bull. Soc. géol. el minéralogique de Brelagne, 1956, fasc. 2, pp. 22-25). 
tion d'une surface d'érosion sur le revers du bloc, surface raccordée à un niveau marin, puis relevée par un bombement ultérieur. Il ne faut pas se dissimuler que cette deuxième solution réclame des coïncidences : pivotement du bloc le long de fractures ayant déjà joué au Miocène ; ajustements, qui restent à déceler, aux régions encadrantes. Rien ne permet d'affirmer que les autres accidents proches, blocs basculés du Mené ou de Gommené, cuvettes de Plessala et de Plémet, aient rejoué pour leur part au Pliocène. S'il n'y a pas là d'empêchement irrésistible à des déformations plus localisées, cette particularité n'en accuse pas moins l'originalité d'une déformation qui parait bien être appelée davantage, en l'état actuel des recherches, par le besoin de prendre un parti que par les faits eux-mêmes.

Une solution de rechange arrangerait tout ; c'est celle qui consisterait à admettre que la transgression pliocène atteignit ici $170 \mathrm{~m}$ sans déformation ultérieure de la surface. Elle poserait, une fois de plus, tout le problème du niveau atteint dans l'Ouest par la mer pliocène et celui des modalités de la sédimentation pliocène. Si l'on veut bien considérer, comme les observations faites sur les dépôts de sables rouges dans la Bretagne centrale et méridionale invitent à le faire, que les dépôts pliocènes n'ont pas fossilisé tout le relief préexistant puisque les cours d'eau retrouvèrent, lors de la régression, leurs anciennes vallées (17), la présence de ces dépôts à $170 \mathrm{~m}$ d'altitude peut être admise. Mais dans la Bretagne centrale et occidentale, si l'on peut déceler un stationnement de la mer pliocène à la cote de $120 \mathrm{~m}$, l'on n'a pas d'autres indications d'un stationnement plus élevé ailleurs que sur le glacis de Merdrignac. Le problème reste donc posé.

\section{CONCLUSION}

Au terme de cette nouvelle étude de la région du Mené, deux points me paraissent établis : la place des reliefs tectoniques tertiaires dans cette contrée, d'une part ; l'appartenance de la crête et du revers occidental du bloc basculé du Mené à la surface éogène, d'autre part.

Mais le problème du glacis méridional reste mal élucidé. Une fois de plus, l'on évolue là dans le brouillard qui enveloppe toujours maints aspects de l'histoire pliocène de la péninsule armoricaine. Les recherches de Mlle Durand ont souvent dissipé cette brume. Elle flotte cependant toujours localement sur cette Bretagne intérieure dont les difficiles problèmes appelleront pendant longtemps des recherches patientes. C'est un recensement détaillé des for-

(17) Voir, en particulier, A. Gurlcher : Le relief de la Bretagne méridionale, Thèse, 1948, p. 562 et M. GauTien : La Bretagne centrale, Thèse, 1947, p. 107. 
mations présumées pliocènes ; c'est l'étude en laboratoire des galets et des sables qu'on y trouve qui doivent permettre de faire la part des dépôts incontestablement pliocènes, de conclure à leur origine marine ou continentale selon les lieux, d'en distinguer les éléments éocènes qui s'y trouvent mêlés, de discerner leurs remaniements ultérieurs dans les terrasses quaternaires. Au fond, c'est souvent le même matériel qui est repris, remanié par des systèmes d'érosion différents (M. Y. Milon l'a depuis longtemps constaté), sur une topographie dont la stabilité tectonique ne fut pas assurée postérieurement au Miocène. Et c'est ce qui fait à la fois la complexité de l'étude et son caractère tentateur. 